\title{
Combinatorial analysis of enhanced green fluorescent protein (EGFP) expression by transplanted hematopoietic stem cells in recipient tissues.
}

\author{
R. P. Visconti*, M. Masuya**, F. Ishikawa**, P. A. Fleming*, M. Ogawa** and C. J. \\ Drake*
}

*Dept. of Anatomy and Cell Biology, Cardiovascular Developmental Biology Center, Medical University of South Carolina, Charleston, SC 29425

** Dept. of Veterans Affairs Medical Center, Department of Medicine, Medical University of South Carolina, Charleston, SC 29425

Recently there have been many reports of the broad tissue engraftment and transdifferentiation potential of bone marrow derived cells. Since these studies made use of crude populations of cells, it is difficult to discern the exact nature of the engrafting cells. To evaluate the transdifferentiation potential of the hematopoietic stem cell (HSC) in vivo, we have used two approaches. One approach employs transplantation of a single enhanced green fluorescent protein (EGFP)-expressing murine HSC (Lin ${ }^{-}, \mathrm{c}^{-\mathrm{kit}^{+}}{ }^{+} \mathrm{Sca}-1^{+}$, $\mathrm{CD}^{-}$) into congenic mice and the other uses xenogenic transplantation of CD $45^{+}$human cord blood cells into SCID mice. Using these transplantation strategies, we have demonstrated that HSC-derived cells reproducibly engraft into a number of recipient tissues, including the kidney, heart and liver. Evaluation of the transdifferentiation potential of these engrafted cells required the use of multiple techniques. Engrafted cells of murine origin were identified by their EGFP expression while those of human origin were detected by fluorescence in situ hybridization (FISH) analysis of human centromeric DNA. To document an unequivocal tissue specific phenotype in these engrafted cells, we combined epifluorescence examination with immunolabeling techniques, RT-PCR and in situ hybridization studies.

Comprehensive examination of these tissues required the use of multiple techniques, including laser scanning confocal microscopy for both en face examination and generation of optical sections, epifluorescence microscopy in conjunction with 
differential interference contrast (DIC) optics to correlate immunolabeling studies with tissue morphology, transmission electron microscopic examination of ultrastructure, and RT-PCR for tissue- and species-specific gene expression.

Additionally, the multiple tissue processing methods required for these studies necessitated development of alternate means for identification of $\mathrm{EGFP}^{+}$cells. These included preservation of fluorescence in paraffin sections, antigen rescue subsequent to tissue processing, anti-GFP immunolabeling in conjunction with FISH analysis and ultrastructural studies (TEM), and detection of EGFP message by in situ hybridization.
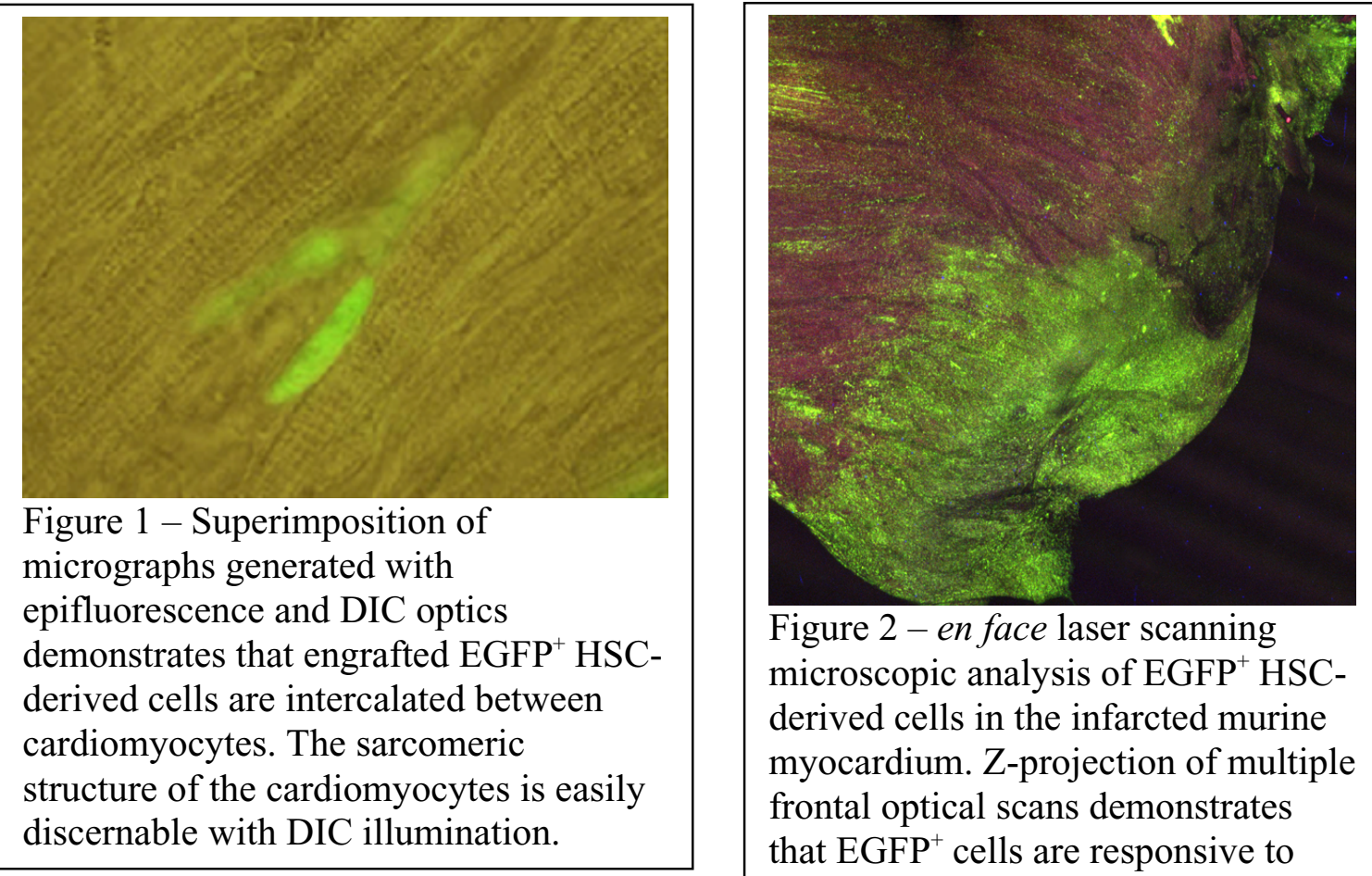

Figure 2 - en face laser scanning microscopic analysis of $\mathrm{EGFP}^{+} \mathrm{HSC}$ derived cells in the infarcted murine myocardium. Z-projection of multiple frontal optical scans demonstrates that $\mathrm{EGFP}^{+}$cells are responsive to hypoxic injury. The increased numbers of $\mathrm{EGFP}^{+}$cells in the myocardial scar make it more intensely green than the adjacent healthy myocardium. 